\title{
Satellite Clusters with Constant Apparent Distribution
}

\author{
David F. Chichka* \\ University of California, Los Angeles, Los Angeles, California 90095
}

\begin{abstract}
The problem of creating a cluster of satellites such that the arrangement maintainsits apparent relative formation as viewed from the planetary surface, without active station keeping, is considered. It is shown that it is possible to create a cluster with nearly constant circular form, but that rotates about its center. The amount of variation from the perfect circle is characterized in terms of the eccentricity of the satellite orbits. Because of the nature of the cluster, it is possible to put a large number of satellites into a cluster, with no danger of satellite collisions. An illustrative example is included.
\end{abstract}

\section{Introduction}

$\mathbf{T}$ HE work begun in this paper was motivated by the problem of creating a cluster of satellites that would maintain a constant, or nearly constant, shape and size when viewed from the Earth. Such a cluster might be said to have constant apparent (as opposed to physical) distribution. In such a formation, all satellites would have a clear field of view of the surface, and would remain in sufficiently close formation to share their information. Such formations are of interest for large distributed-aperture sensing, for example. Another possibility is that of forming clusters from many small, inexpensive satellites, each with a particular type of sensor and some computing power. Remaining in a close formation would allow the satellites to share information and computing ability among themselves.

For such missions, it might be unnecessary to maintain extremely precise relative positions; rather, it might be sufficient to know the relative position accurately, and to remain in close enough proximity to allow intercraft communication. In addition, an effect of constant apparent distribution is that the angular dispositions of the satellites relative to their ground targets and each other would be constant.

One of the desires of this work is to reduce the need for stationkeeping thrust. For this reason, we consider orbits in which the natural motion of the satellites keeps them in a cluster. The need for station-keeping would then be reduced to eliminating the effects of perturbations on the array. These effects will be found in the bulk motion of the array and in the relative motions of the satellites in the array. For missions in which precise knowledge of position is the primary goal, some cyclic perturbations may be acceptable if they are sufficiently small and well understood.

In the next section, we will examine the problem using the wellknown Clohessy-Wiltshire $(\mathrm{C}-\mathrm{W})$ linearized equations. ${ }^{1}$ This will produce a result valid for a very small cluster. In the third section, we will use classical orbital parameters to quantify the errors in the linear approximation. An example is then included.

While developing the ideas in this paper, the author became aware of the work of DeCou, ${ }^{2}$ who examined the problem of satellite interferometry. This special case limits the cluster to three colinear satellites, reducing them to the same circular orbit for the problem considered here. More general study of interferometry in solar orbit is reported in Refs. 3 and 4. Several other authors have also addressed the problem of widely spaced satellite constellations (see, for example, Ulybyshev, ${ }^{5}$ McInnes, ${ }^{6}$ and the references therein). More recently, some results of linear cluster analysis have been reported by Sedwick et al. ${ }^{7}$

Received 13 October 1998; revision received 7 March 2000; accepted for publication 15 May 2000. Copyright (C) 2000 by the American Institute of Aeronautics and Astronautics, Inc. All rights reserved.

*Assistant Research Engineer, Mechanical and Aerospace Engineering Department, 420 Westwood Plaza; also Senior Engineer, Control and Dynamical Systems Department, California Institute of Technology, Pasadena, CA 91125. Member AIAA.

\section{Notation and Problem Description}

In what follows, the motion of a satellite will be described with respect both to a moving reference point and as seen by an observer on the surface of a spherical planet. The motion of the reference point can be visualized by thinking of it as the position of a (possibly hypothetical) referencesatellite on a circularorbit of radius $R$, which will be referred to as the reference orbit. This reference point will serve as the origin of a local coordinate system. This referenceframe was first described by Hill, ${ }^{8}$ who derived it in his work on the motion of the moon about the Earth.

Let $\boldsymbol{R}$ be the position of the reference satellite, following the reference orbit under idealized two-body motion, and $\boldsymbol{r}$ the position of a nearby point. Both $\boldsymbol{R}$ and $\boldsymbol{r}$ are expressed in an inertial frame centered at the planetary center of mass. Hill's coordinate frame moves with the reference point, and rotates such that the $x$ axis is aligned with $\boldsymbol{R}$. The $y$ axis is tangent to the reference orbit at the reference point, and positive in the direction of the orbital motion. The $z$ axis completes the orthogonal set, as illustrated in Fig. 1. Thus, the reference point is also the origin of the moving reference frame.

Hill's frame allows the motion of the actual satellite to be described with respect to the reference point. It will be shown that the motion of the satellite in this frame will create an orbit; this orbit will be called the relative orbit, as shown in Fig. 1. We are primarily interested, however, in the relative motion as it appears to a fictional observer on the planetary surface, whose position is always on the ray connecting the center of the planet to the reference point. This observer represents the point on the surface that is the subject of observation by the satellite cluster.

To this end, we define the apparent orbit. Consider a point on the planetary surface that moves with time such that it is always between the center of the planet and the origin of Hill's frame. The apparent orbit is the motion of the satellite relative to the reference point as seen from this point. As this is purely a matter of the line of sight from the viewer to the satellite, there is no physical meaning to the apparent orbit. However, it is helpful to visualize it as the trace left by the intersection of the line of sight as it passes through the $y-z$ plane in the Hill's coordinate frame, as shown in Fig. 2 .

The analysis that follows will be primarily concerned with three angles that describe the position of the satellite on the apparent orbit. The elevation angle $\kappa$, the lateral angle $\lambda$, and the rotary angle $\phi$ are shown in Fig. 3. The angles are all positive as shown, with $\phi$ increasing in right-hand rotation about the Hill's-frame $x$ axis.

The maximum value of $\lambda$ will be referred to as the angular width of the orbit, and the maximum value of $\kappa$ as the angular height. $R_{P}$ will denote the radius of the planet, and the value $R-R_{P}$ will be called the altitude of the reference orbit. This is also the mean altitude of the satellite cluster.

Remark 1: A more rigorous definition of the apparent orbit would be the trace of the line of sight as it passes through a spherical shell centered at the viewpoint, with radius $R-R_{P}$. Using this definition, a perfectly circular apparent orbit would result in a constant total angle between the line of sight and the line joining the viewpoint 


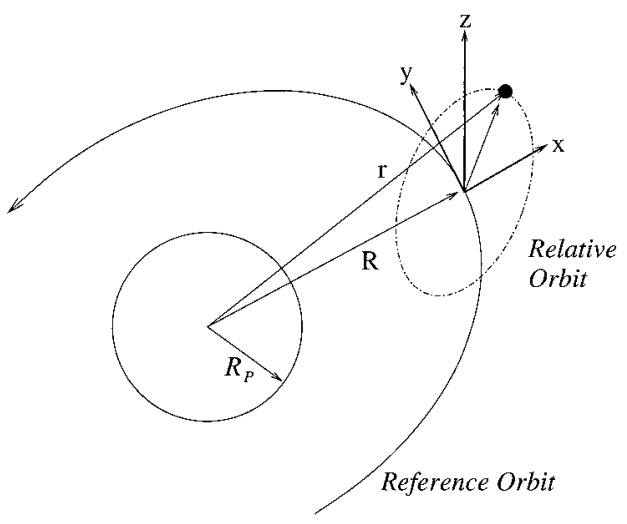

Fig. 1 Hill's reference frame for satellite relative motion.

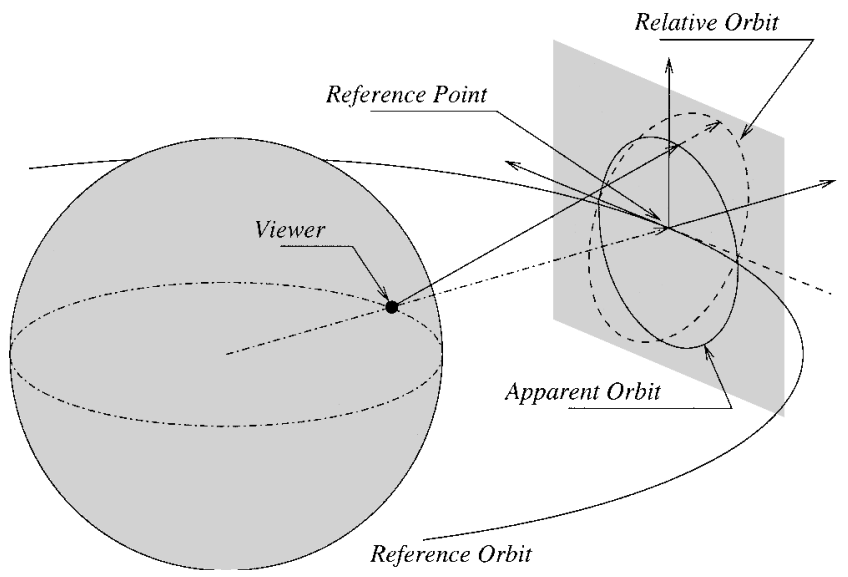

Fig. 2 Reference, relative, and apparent orbits.

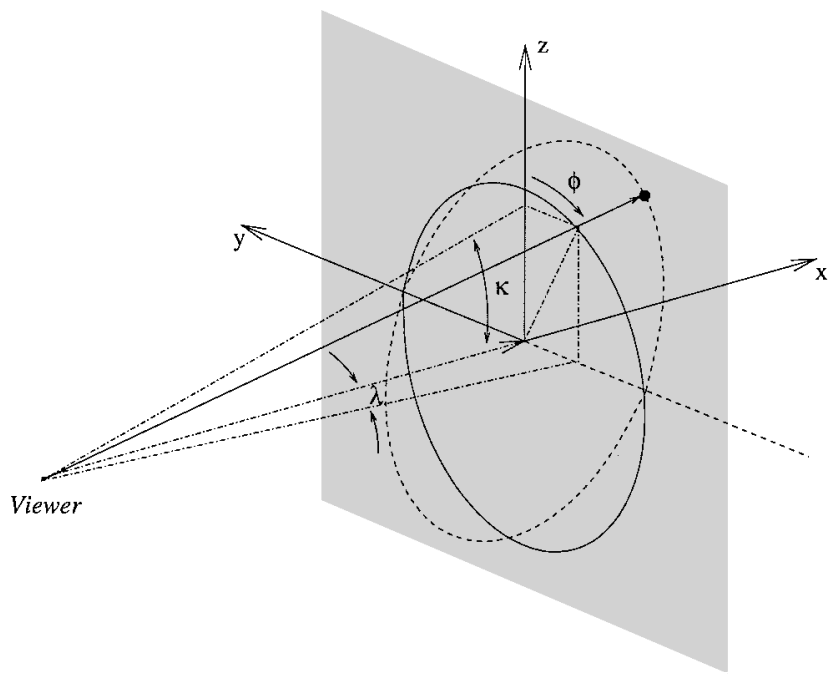

Fig. 3 Geometry description and definitions.

to the origin of the Hill's frame. This will not be important in what follows, as the analysis will be done by considering the angles themselves, rather than projected distances. The apparent orbit can then be calculated by multiplying the angles by $R-R_{P}$. The difference in this formal method and the visualization suggested above is extremely small for clusters in which the cluster radius is small with respect to its mean altitude.

The symbols $a$ and $e$ will denote the semimajor axis and eccentricity of an orbit. The variable $n$ is the mean motion or average angular motion of a body on an orbit, and for an elliptic orbit is given by the relation $n=\sqrt{ }\left(\mu / a^{3}\right)$, where $\mu$ is the gravitationalparameter of the central body (the mass of the planet times the universal gravitational constant). The true anomaly $v$ of a point on an eccentric orbit

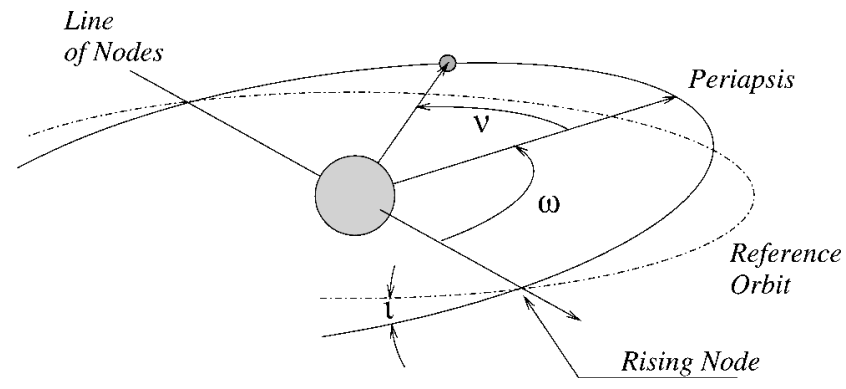

Fig. 4 Orbital elements of the eccentric orbit.

will be measured from periapsis, as shown in Fig. 4. Later sections will consider an eccentric orbit, inclined with respect to a circular reference orbit. In this case, the reference plane will be the plane of the circular orbit, and the line of nodes will be taken to be the line of intersection of the two orbit planes. The argument of periapsis $\omega$ of the eccentric orbit will be measured from this line of nodes. The inclination will be denoted $\imath$ and will be the angle between the two orbit planes. Finally, the angle $u=\omega+v$ is the angle from the line of nodes to the radial vector. Note that this is defined for circular as well as eccentric orbits.

\section{Linearized Approach}

The $\mathrm{C}-\mathrm{W}$ equations are a natural choice for describing the motion of a satellite near a circularreference orbit. They are defined in Hill's reference frame as in Fig. 1, and are sometimes referred to as Hill's equations. The equations are written

$$
\begin{aligned}
& \ddot{x}=3 n^{2} x+2 n \dot{y} \\
& \ddot{y}=-2 n \dot{x} \\
& \ddot{z}=-n^{2} z
\end{aligned}
$$

where $n=\sqrt{ }\left(\mu / R^{3}\right)$ is the angular rate of the reference orbit. It follows immediately that the out-of-plane motion is given by

$$
z(t)=A_{z} \cos \left(n t+\phi_{z}\right)
$$

where $A_{z}$ is the magnitude and $\phi_{z}$ is a constant phase shift. Solving for $x$ and $y$ is only slightly more difficult. Following usual practice, integrate $y$ to get

$$
\dot{y}=-2 n x+k
$$

where $k$ is a constant of integration. Subsitute this into the $x$ equation to get

$$
\ddot{x}=-n^{2} x+2 n k
$$

This is directly solved to get

$$
x(t)=A_{x} \cos \left(n t+\phi_{x}\right)+2 k / n
$$

which is substituted into Eq. (5) and integrated to get

$$
y(t)=-2 A_{x} \sin \left(n t+\phi_{x}\right)-3 k t+c
$$

where $c$ is the constant of integration.

Because we require a purely cyclic motion with the same period as the reference orbit for our problem, $k=0 ; c$ is simply an offset term and can be given the value zero without loss of generality.

We are concernedonly with the orbit as it appears from the surface of the planet. In keeping with the assumptions made to linearize the equations of motion, we assume that the variation in $r$ is negligible with respect to the altitude of the orbit. The appearance of the orbit will then be its projection on the $y-z$ plane. We have

$$
\begin{gathered}
y(t)=-2 A_{x} \sin \left(n t+\phi_{x}\right) \\
z(t)=A_{z} \cos \left(n t+\phi_{z}\right)
\end{gathered}
$$




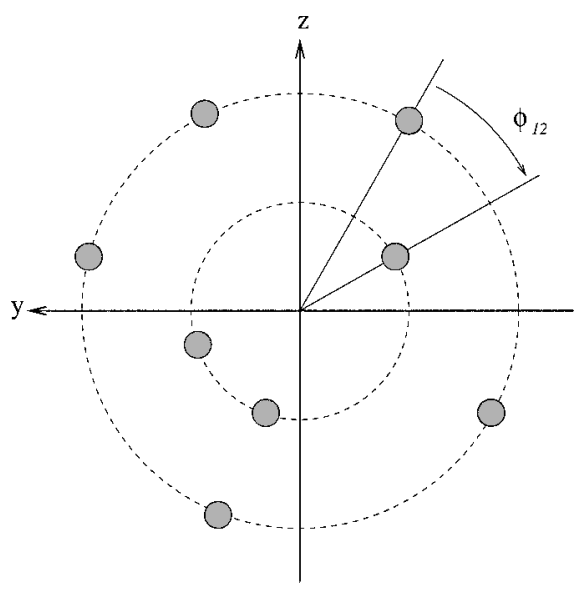

Fig. 5 Apparently circular cluster of satellites.

Setting $\phi_{x}=\phi_{z}$ results in a perfectly elliptic projection. By properly specifying the initial conditions, it is possible to set $A_{z}=2 A_{x}$ and the apparent orbit becomes a circle. (Note that setting $\phi_{x}=\phi_{z}+\pi$ also results in an elliptic projection, with the direction of the projected motion reversed.)

Remark 2: The statement that the apparent orbit is the $y-z$ projection assumes that the viewpoint is on the line connecting the center of the planet to the center of the relative orbit. Extension to the case of a viewpoint above or below this line is straightforward, so long as the viewpoint remains on the plane normal to the plane of the reference orbit.

Because the initial angle $\phi_{z}$ in Eq. (9) is arbitrary, it is possible to place as many satellites in a circular apparent orbit as desired, each separated from the next by some constant angle. This would give a "pinwheel" effect from the planetary surface as the satellites rotate about the center of the cluster. The cluster would spin about its own center once during each orbit of the planet.

The radius of the apparent orbit is also arbitrary. Thus, it is possible to create a cluster of an almost arbitrary number of satellites, by creating concentric rings about the reference satellite, as in Fig. 5. The period of the apparent orbit is always that of the reference orbit, so that the entire cluster will maintain its shape relative to the center. Thus, separation angles such as $\phi_{12}$ in Fig. 5 should remain constant.

As the $\mathrm{C}-\mathrm{W}$ equations are linearizations, it is to be expected that they do not fully describe the relative motion of the satellite. An approximation of their error can be made by examining the higherorder terms in the orbital equations. This is the subject of the next section.

Remark 3: Note that the height of the apparentorbit is independent of the width. Thus, whereas this paper is concerned only with the apparent relative orbit, this technique can also be used to generate a relative orbit that is very nearly circular in actuality, and which appears elliptic when viewed from the planetary surface.

\section{Orbital Mechanics}

The motion of the satellite described by the $\mathrm{C}-\mathrm{W}$ equations is caused by slight differences in the orbital parameters of the satellites. The satellite in the elliptic orbit moves more slowly than the reference when at apoapsis, and more quickly near periapsis. It thus seems to lag behind and then to catch up. When combined with inclination, this leads to an apparent orbit about the reference satellite.

Investigating the apparent orbit in terms of the orbital elements is not as straightforwardas using the linear equations, but this approach allows an investigation of the limits of the linearized approach, and an estimate of the corrections due to nonlinearity. In this section, we will investigate the eccentricity and inclination required to produce a circular apparent orbit.

In developing the ideas of this section, we will confine our attention to orbits in which periapsis is $90 \mathrm{deg}$ from the line of nodes. This ensures the symmetry of the apparent orbit about the Hill's-frame $z$ axis. (Recall that we are defining the line of nodes as the line of intersection of the orbital plane with the reference plane.)
The description of the apparent orbit will be expressed in terms of the eccentricity of the satellite orbit. These terms will be on the order of $e$; thus, second-order terms in $e$ may be considered firstorder corrections. In the following analysis, we will find estimates for these correction terms.

\section{A. Apparent Angular Width}

We first examine the eccentricity needed to produce an apparent orbit of a particular width. The width will be defined by projecting the line of sight to the satellite onto the reference plane. The angle between this projection and the line of sight to the reference point is then the angular separation of the satellite and the reference point (this is the angle $\lambda$ in Fig. 3). The width of the orbit is then the maximum value of this angle.

If the inclination of the orbit is zero, the angle is easily computed. We take the reference orbit and the eccentric orbit to have equal semimajor axes and thus equal periods. The motion of the reference point is then equivalent to the mean motion of the eccentric orbit. The separation at any point is then the difference between the true anomaly $v$ and the mean anomaly $M$. This is easily addressed, as the expansion of $v$ in terms of $M$ is well known. Assuming the inclination is small enough that its effects are negligible then allows us to extract some information quickly. The expansion as given in Moulton ${ }^{9}$ is

$$
v-M=2 e \sin M+\frac{5}{4} e^{2} \sin 2 M+\cdots
$$

Finding the maximum is then a matter of simple calculus. Taking the expansion through second order and taking the derivative with respect to $M$ results in

$$
4 e \cos M+5 e^{2} \cos 2 M=0
$$

For $e \rightarrow 0$, this leads to values of $M$ approaching $\pi / 2$, agreeing with intuition. Setting $M=\pi / 2+\delta$ and dividing through by $e$ allows Eq. (11) to be rewritten as

$$
-2 \sin \delta-5 e \cos 2 \delta=0
$$

Again, $e=0$ gives $\delta=0$, and for very small $e$ we have that $\delta$ is approximately $-5 e / 2$. The sign of $\delta$ is less important than the knowledge that $\delta$ is of order $e$. This will make obvious the order of terms in the analysis to follow.

Using these results, return to Eq. (10). This equation can now be written

$$
v-M=2 e \cos \delta-\frac{5}{4} e^{2} \sin 2 \delta+\cdots
$$

Because $\delta$ is small, $\cos \delta \sim 1$ and $\sin 2 \delta \sim 2 \delta$. Because $\delta$ is of order $e$, the second term on the right in the above equation is $\sim e^{3}$ and can be ignored. Thus, to second order,

$$
v-M=2 e
$$

Thus, the angular width of the apparent orbit as seen from the center of the central body is $4 e$. From the surface of the planet, the angular spread is wider. Letting $\lambda$ denote the angle between the lines of sight to the satellite and to the reference, geometry gives

$$
\lambda=\tan ^{-1}\left(\frac{r \sin (v-M)}{r \cos (v-M)-R_{P}}\right)
$$

Expanding the radius of the elliptic orbit in $M$ and $e$, again writing $M$ as $\pi / 2+\delta$, gives

$$
r=R\left[1-e \sin \delta+\left(e^{2} / 2\right)(\cos 2 \delta-1)+\cdots\right]
$$

Using the expansions for $v-M$ and $r$ as given, we find that to first order

$$
\lambda=\frac{2 R \cos \delta}{R-R_{P}} e \approx \frac{2 R e}{R-R_{P}}
$$


and that the coefficient of the second-orderterm is of order $\delta$, making the term of third order. This gives a total angular width of the apparent orbit of

$$
2 \lambda \approx 4 e\left[R /\left(R-R_{P}\right)\right]
$$

For a low-altitude orbit, $R_{P}$ may be nearly as large as $R$, and thus the angular spread of the apparent orbit as seen from the planetary surface might be several times larger than the value of $v-M$.

\section{B. Correction for Inclination}

To allow for orbital inclination, the expansions used to compute the width of the apparent orbit must be extended to include the inclination. Consider the angle between the projection of the radial vector onto the reference plane and the projection of the eccentricity vector (that is, the vector from the center of the planet to periapsis). Letting this angle be denoted $\nu$, we have

$$
\nu=\tan ^{-1}\left(\frac{r \sin v}{r \cos v \cos \imath}\right)=\tan ^{-1}\left(\frac{\tan v}{\cos \imath}\right)
$$

The width of the apparent orbit is then the difference between this angle and the mean anomaly $M$ of the reference orbit.

We expect that this angle will be maximized near $v=\pi / 2$. Because we have assumed that the argument of periapsis $\omega=\pi / 2$, this corresponds to the descending node. Expanding about this point, we define the small parameter $\varepsilon=v-\pi / 2$, and the angle corresponding to $\nu$ as

$$
\tan \varepsilon=\frac{r \sin \varepsilon \cos \imath}{r \cos \varepsilon} \Rightarrow \varepsilon=\tan ^{-1}[\alpha \tan \varepsilon]
$$

where for brevity we use $\alpha$ in place of $\cos t$. This angle is measured from the line of nodes to the projection of the radial vector. We expect that $\varepsilon \approx 0$, so that Eq. (18) should be valid over the area of interest.

The expansion for $\varepsilon$ is straightforward:

$$
\varepsilon=\alpha \varepsilon+\left[\left(\alpha+\alpha^{3}\right) / 3\right] \varepsilon^{3}+O\left(\varepsilon^{5}\right)
$$

The angular width is found by introducing the expansion for $v$ into this expression. At this point, we make the assumption that our earlier results were "nearly" correct, and thus that $\delta$ is in fact of about the same size as $e$ and that $\imath \approx c e$ for some constant $c$ at least through first order.

Knowing that $\delta$ is of the order of $e$ allows us to ignore all terms above third order combined in the two variables. Thus, we achieve

$$
\begin{aligned}
\varepsilon-\delta & =(\alpha-1) \delta+\left(1-\alpha^{2}\right) \alpha \delta^{3} / 3+2 \alpha \cos \delta\left[1+\left(1-\alpha^{2}\right) \delta^{2}\right] e \\
+ & {\left[16\left(1-\alpha^{2}\right) \delta \cos ^{2} \delta-5 \sin 2 \delta\right](\alpha / 4) e^{2}+\left[32\left(1-\alpha^{2}\right) \cos ^{3} \delta\right.} \\
& -3 \cos \delta-13 \cos 3 \delta](\alpha / 12) e^{3}+\cdots
\end{aligned}
$$

Substituting $\cos \imath=\cos (c e)$ for $\alpha$ and expanding the trigonometric terms gives the expansion as

$$
\varepsilon-\delta=2 e-\delta^{2} e-\left(5+c^{2}\right) \delta e^{2} / 2-\left(c^{2}+4 / 3\right) e^{3}
$$

through third order combined. Through first order, we again have the width to be $2 e$, and we see once more that there are no second-order terms.

\section{Apparent Vertical Size}

The apparent vertical size of the orbit is determined by the elevation angle between the line of sight to the satellite and the reference plane. Relative to the center of the planet, this angle can be expressed as

$$
\kappa_{c}=\sin ^{-1}[\sin u \sin t]
$$

where $u$ is the angle from the line of nodes to the radial vector. As a result of our requirement on the argument of periapsis, the absolute value will be maximized for $u= \pm \pi / 2$. For a circular orbit, we require that the maximum value of $\kappa_{c}$ equal the maximum value of $v-M$. Thus, from Eq. (13) we have

$$
\kappa_{c}=\sin ^{-1}(\sin \imath)=\imath=v-M=2 e
$$

to second order in $e$.

The vertical angle as seen from the surface of the planet is widened, as is the lateral. However, as the maximum vertical spread occurs at the extrema of the radius, there is a first-order contribution of $e$ to $r$ that must be examined.

When the satellite is at the apses, the apparentelevation angle can be written as

$$
\kappa=\tan ^{-1}\left(\frac{R(1+\varepsilon) \sin \imath}{R(1+\varepsilon) \cos \imath-R_{P}}\right)
$$

where $\varepsilon$ is equal in magnitude to the eccentricity, and is positive at apoapsis and negative at periapsis. We expand about $\varepsilon=0$ to get

$$
\kappa=\tan ^{-1}\left(\frac{R \sin \imath}{R \cos \imath-R_{P}}\right)-\frac{R R_{P} \sin \imath}{\left(R-R_{P}\right)^{2}+R^{2} \sin ^{2} \imath} \varepsilon+\cdots
$$

Recalling Eq. (22) and making small angle approximations, the first term reduces to

$$
\kappa \approx 2 \operatorname{Re} /\left(R-R_{P}\right)
$$

as would be expected. The second term is the first-order correction to $\kappa$.

Note that the coefficient of the correction contains $R$ and $R_{P}$. We make the assumption that the product $\operatorname{Re} /\left(R-R_{P}\right)$ is small. This is of concern only when $R_{P}$ approaches $R$, as for a low-altitude orbit. In this case, it is seen that the correction term is of second order in $\operatorname{Re} /\left(R-R_{P}\right)$.

The coefficient of the correction term is negative, showing an expansion of the angle at periapsis and a contraction at apoapsis. The effect of this could be to raise the apparent orbit slightly with respect to the reference plane. If in fact the apparentorbit is elevated, there should be a corresponding elevation at the points of maximum lateral spread.

To investigate this, we require a value for the position of the satellite on its orbit at which the apparent orbit achieves it maximum width. In an attempt to find this value, we can return to Eq. (20). If we take the derivative of this equation, however, we find no terms that are of first order combined. Because the value of $\varepsilon-\delta$, and thus of $l$, is defined only through second order, first-order terms are necessary to obtain any information. Numerical investigations show that the elevation of the point of maximum width is in fact not linear with $e$.

\section{Phase Separation in Apparent Orbit}

When there is more than one satellite in apparent orbit about the reference point, they will be separated by some angle, constant to the accuracy of the C-W equations. In Fig. 5, the angle denoted $\phi_{12}$ is such a separation angle.

The separation angle between two satellites in the same apparent orbit is a function of the angle between the lines of nodes of their orbits. Nominally, the separation angle equals this angle. The separation will vary as a result of the second-order deviations of the apparent orbit from perfectcircularity; the actual amount of variance will be dependent upon the separation.

Although this makes it impossible to define the change in separation angle, we can investigate the angular rate of the satellite in its apparent orbit. It is clear that the satellite will sweep its angle faster when it is at periapsis than at apoapsis; the ratio will be the same as that for the true anomaly rates:

$$
\dot{\phi}_{p} / \dot{\phi}_{a}=[(1+e) /(1-e)]^{2}
$$

where $\phi$ is the phase angle in the apparent orbit and the subscripts refer to periapsis and apoapsis.

An estimate of the total variation in $\phi(t)$ from that predicted by the $\mathrm{C}-\mathrm{W}$ equations can be had by finding the value of the true 
anomaly when the mean anomaly equals $\pi / 2$. This is easily enough accomplished through the same expansion used earlier:

$$
v=M+2 e \sin M+\left(5 e^{2} / 4\right) \sin 2 M+\cdots \Rightarrow v=\pi / 2+2 e
$$

through second order. The phase angle in the apparent orbit, measured from the vertical, can be expressed approximately as

$$
\phi(M=\pi / 2)=\frac{\pi}{2}+\tan ^{-1}\left(\frac{r \sin u \sin \imath}{R(v-M)}\right) \approx \pi / 2+\sin \imath
$$

Recalling that $\imath \approx 2 e$, this implies that the satellite sweeps through $4 e$ less apparent angle as $M$ goes from $\pi / 2$ to $3 \pi / 2$ than during the other half of the orbit. Thus, two satellites in orbit about the reference, separated by a 180 -deg difference in phase, will vary in their relative positions by $\pm 4 e$ radians during the orbit.

\section{E. Geometry of the Relative Orbit}

The apparent orbit with which we have been concerned is in a sense the projection of the relative orbit onto a plane normal to the radius of the reference orbit. The actual relative orbit is not circular. Rather, it is nearly elliptical. This follows from the results of Sec. III.

The depth of the motion is clearly the differencebetween periapsis and apoapsis, which by definition is $2 R e$. As the first-order approximation of the diameter of the apparent orbit is $4 R e$, this implies that the relative motion lies near a plane that is angled $60 \mathrm{deg}$ with respect to the reference plane. As a result of this, the variation in the actual distance from the reference point to the satellite will vary from a minimum of the apparent orbital radius (when the satellite crosses the reference plane) to a maximum of approximately $\sqrt{ }(5) / 2$ times this amount (at periapsis and apoapsis). Thus the actual maximum distance will be (to first order in $e) \sqrt{ }(5)\left(R-R_{P}\right) e$.

\section{Example}

We consider here an example to demonstrate numerically the effects of the correction terms on the linearized solution. As a difficult case, we will consider a fairly large apparent orbit at a low altitude.

As has been mentioned in the preceding section, the appearance of the relative orbit is a matter of the angles between the lines of sight to the satellite and to the reference, from a point on the planetary surface along the vector joining the center of the planet to the reference. To speak of the radius of the apparent orbit requires a more precise definition. In this case, we will simply multiply the angles describing the relative orbit by the altitude of the reference orbit.

Consider a cluster of Earth satellites, such as shown in Fig. 6, in apparent orbit about a central point. The nominal orbital altitude is $552 \mathrm{~km}(298 \mathrm{nmi})$. To exaggerate the second-order effects, we will set the radius of the apparent orbit at $10 \mathrm{~km}$. We assume a spherical Earth. The cluster consists of a single ring, with six satellites equally spaced such that their nominal apparentangularseparation is $60 \mathrm{deg}$.

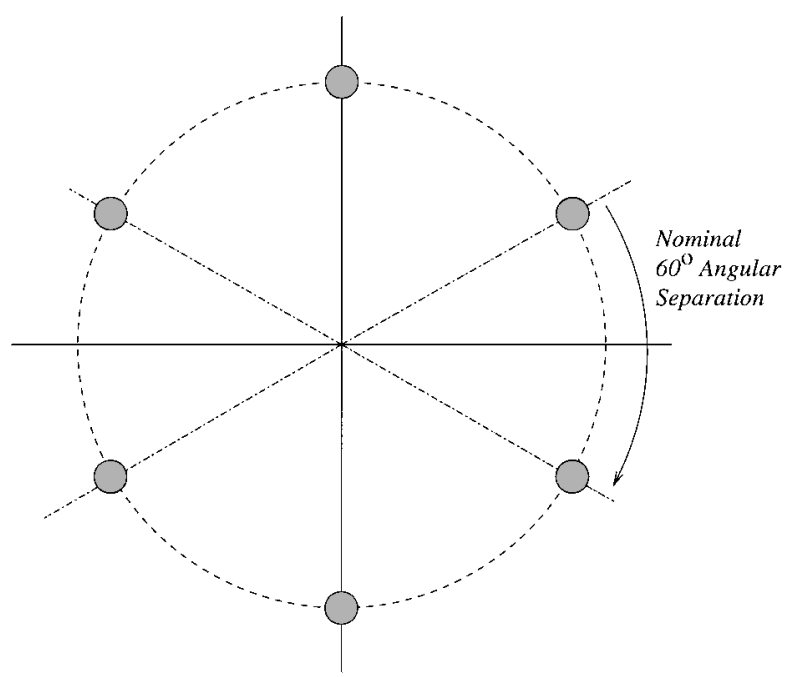

Fig. 6 Ring of six equally spaced satellites.
Using the results derived above, we have that the angular spread of the desired orbit is $\lambda=10 / 552=0.0181$ rad. From Eq. (16) we then have that

$$
0.0181=2 \operatorname{Re} /\left(R-R_{P}\right) \Rightarrow e=7.21 \times 10^{-4}
$$

The inclination angle equals the spread angle, so that $t=2 e=$ $1.44 \times 10^{-3}=0.0827 \mathrm{deg}$. The first-ordercorrectionsto the vertical spread of the orbit are

$$
\Delta \kappa=0.209 e=1.51 \times 10^{-4}=0.00865 \mathrm{deg}
$$

which when multiplied by the nominal altitude of the orbit produces an apparent shift of $83.34 \mathrm{~m}$.

The shape of the apparent orbit is so close to circular that it appears to be so to the naked eye. The widest point is reached at $t / P=0.2484$, and the value of $v-M$ at this point is $1.99992 e$. The width as viewed from the surface of the Earth is $9.9995 \mathrm{~km}$, an error due to higher-order terms of less than one meter.

Figure 7 shows the errors in vertical and horizontal positions, again in meters. The vertical error is primarily above the zero axis, reflecting the vertical shift in the apparent orbit discussed in the

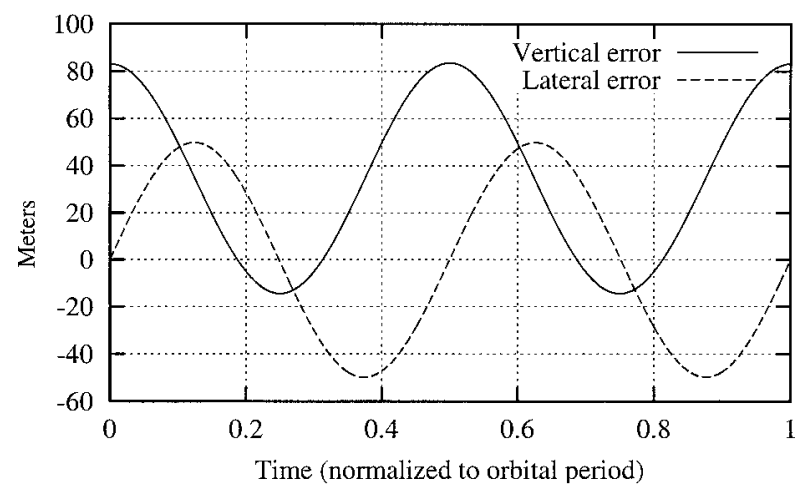

Fig. 7 Apparent position variations from nominal.

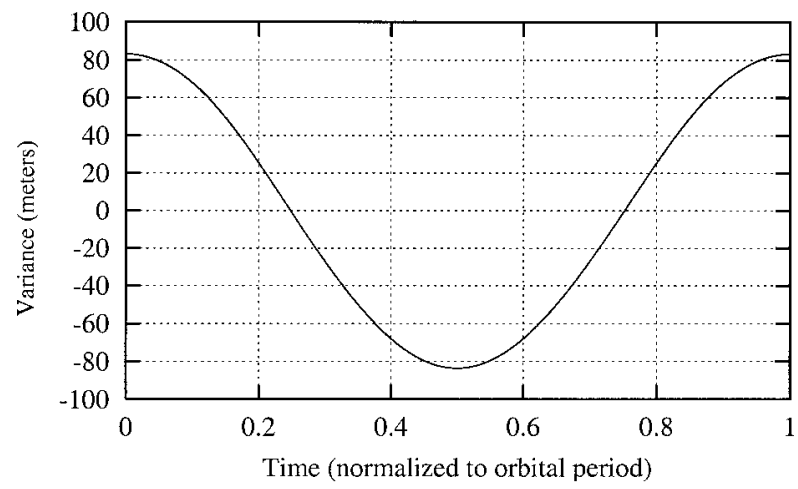

Fig. 8 Variation in radius of apparent orbit from nominal.

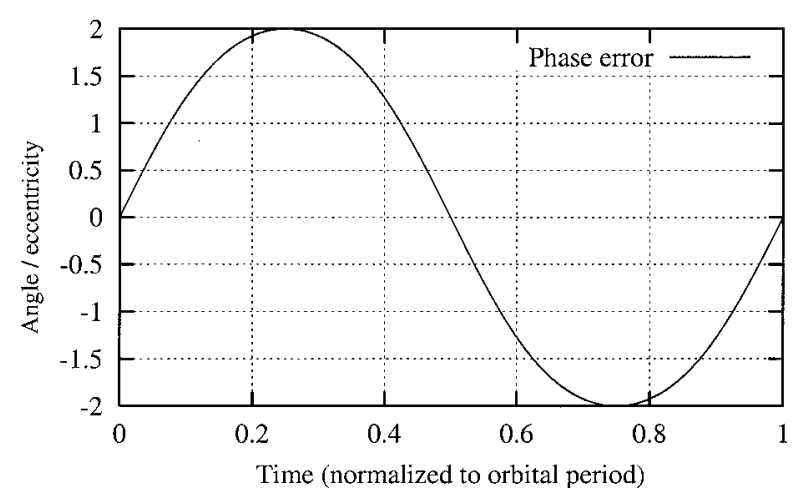

Fig. 9 Error in angle in apparent orbit, normalized to eccentricity 


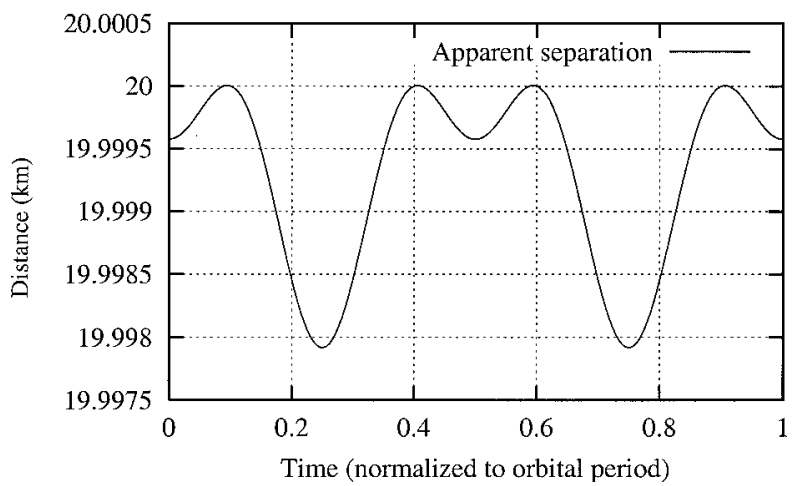

Fig. 10 Apparent distance between opposed satellites.

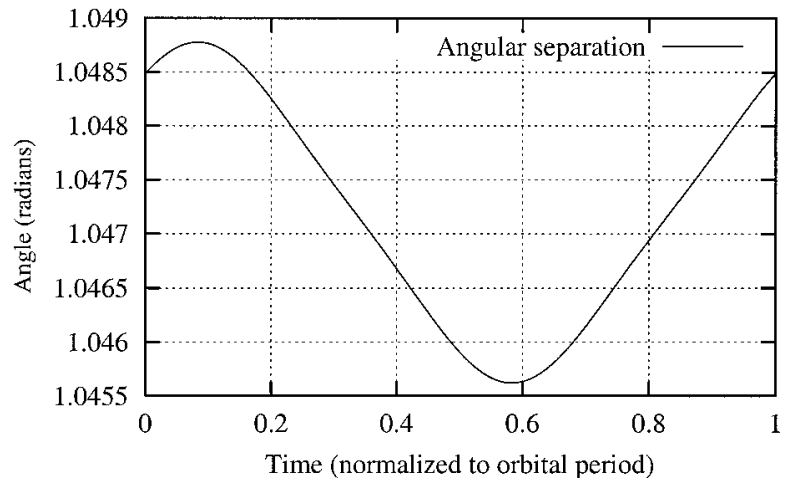

Fig. 11 Angle between neighboring satellites in cluster of six.

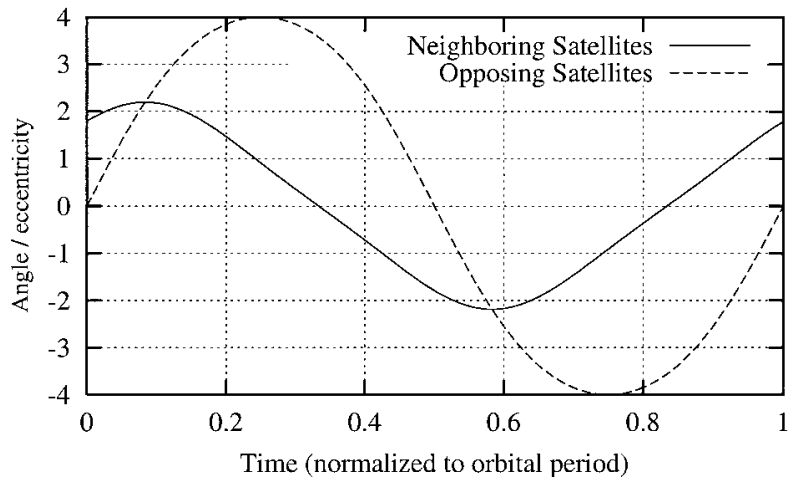

Fig. 12 Errors in relative angle in cluster of six satellites.

preceding paragraphs. The vertical error is negative, however, in the neighborhood of $t / P=0.25$.

Figure 8 shows the variation in the distance from the reference point to the satellite, in meters; the maximum value is $83.13 \mathrm{~m}$, within $0.22 \mathrm{~m}$ of the value predicted by the first-order correction term. Figure 9 shows the difference between the calculated angular position in the apparent orbit and the ideal. The ideal value of the angle is simply $2 \pi t / P$. The displayed error value is divided by the eccentricity. It is seen that the maximum value is very close to the predicted value of $2 e$.

The apparent distance between two satellites on opposite sides of the apparent orbit is shown in Fig. 10. The distance is constant to within $2.2 \mathrm{~m}$; the variation from nominal for one satellite is almost perfectly matched by an equal variation for the other.

The angle between two neighboring satellites in the cluster of six is shown in Fig. 11. Here, it is seen that the angle is variable by approximately $\pm 0.09 \mathrm{deg}$. This is about $2.18 e$, well within the predicted $\pm 4 e$. This is made more explicit in Fig. 12, in which this error is displayed normalized to the eccentricity. Also shown in Fig. 12 is the variation of the angle between two opposing satellites in the cluster. Here we see precisely the $-4 e$ to $4 e$ cycling predicted.

\section{Conclusions}

This paper looks at the possibility of creating satellite clusters that through their natural motion retain a constant shape when viewed from a planet's surface. It is shown that the shape of the apparent circular orbit can be described in terms of the eccentricity of the orbit, and that terms through second order in $e$ are sufficient to describe the motion to a high order of accuracy. An example is included to demonstrate the accuracy of the analysis.

The analysis of this paper considers only viewpoints that are on the line connecting the center of the planet to the origin of the moving reference frame. It is also limited by the assumption of a spherical planet; in considering satellite clusters about the Earth, the effects of nonsphericity must be included. These efforts are the subject of continuing work.

\section{Acknowledgment}

The author wishes to thank D. L. Mingori of the University of California, Los Angeles, for several helpful comments and conversations.

\section{References}

${ }^{1}$ Prussing, J. E., and Conway, B. A., Orbital Mechanics, Oxford Univ. Press, New York, 1993, pp. 139-149.

${ }^{2}$ DeCou, A. B., "Orbital Station-Keeping for Multiple Spacecraft Interferometry," Journal of Astronautical Sciences, Vol. 39, No. 3, 1991, pp. $283-$ 297.

${ }^{3}$ Vincent, M. A., and Bender, P. L., "Orbital Mechanics of a Space-Borne Gravitational Wave Experiment," American Astronautical Society Paper AAS 87-523, Springfield, VA, Aug. 1987.

${ }^{4}$ Folkner, W. M., Bender, P. L., and Stebbins, R. T., "LISA Mission Concept Study: Laser Interferometry Space Antenna for the Detection and Observation of Gravitational Waves," Jet Propulsion Lab., Publ. 97-16, California Inst. of Technology, Pasadena, CA, March 1998.

${ }^{5}$ Ulybyshev, Y., "Long-Term Formation Keeping of Satellite Constellation Using Linear-Quadratic Controller," Journal of Guidance, Control, and Dynamics, Vol. 21, No. 1, 1998, pp. 109-115.

${ }^{6}$ McInnes, C. R., "Autonomous Ring Formation for a Planar Constellation of Satellites," Journal of Guidance, Control, and Dynamics, Vol. 18, No. 5, 1995, pp. 1215-1217.

${ }^{7}$ Sedwick, R. J., Kong, E. M. C., and Miller, D. W., "Exploiting Orbital Dynamics and Micropropulsion for Aperture Synthesis Using Distributed Satellite Systems-Applications to TechSat21," AIAA Paper 98-5289, Oct. 1998.

${ }^{8}$ Hill, G. W., "Researches in the Lunar Theory," American Journal of Mathematics, Vol. 1, 1878, pp. 5-26.

${ }^{9}$ Moulton, F. R., An Introduction to Celestial Mechanics, 2nd rev. ed., Dover, New York, 1970. 